\title{
Left Ventricular Ejection Fraction Measured with Doppler Color Flow Mapping Techniques
}

\author{
A. Resai Bengur, MD, A. Rebecca Snider, MD, Roger P. Vermilion, MD, and John C. Freeland
}

To determine if left ventricular (LV) ejection fraction (EF) can be accurately measured from the color Doppler examination, 11 patients (aged 0.4 to 22 years) underwent 2 -dimensional and color Doppler examinations within 24 hours of cardiac catheterization. With use of a biplane Simpson's rule, LV end-diastolic volume, endsystolic volume and EF were measured from cineangiograms, 2-dimensional echocardiograms and color Doppler examinations. The 2-dimensional echocardiographic and color Doppler measurements were obtained from apical 4-chamber and long-axis views. The color Doppler examinations were performed by placing the color sector over the left ventricle only. The velocity scale was set at the lowest possible Nyquist limit $(<0.17 \mathrm{~m} / \mathrm{s})$, and the highest possible carrier frequency was used to obtain this limit. With these settings, all flow signals in the LV chamber were aliased so that the entire chamber was filled with mosaic color Doppler signals. Motion of the surrounding LV walls gave rise to nonaliased (pure red-blue) signals. With use of an off-line analysis system equipped with a color frame grabber, the border of the mosaic color flow area was traced to obtain volumes and EF. End-diastolic and endsystolic volumes measured with color Doppler correlated well with those measured from 2-dimensional echocardiography $(r=0.99$, standard error of the estimate [SEE] = $11.9 \mathrm{ml} r \mathrm{r}=0.99$, SEE $=4.4 \mathrm{ml}$, respectively) and cineangiography ( $r=0.92$, SEE $=16.8 \mathrm{ml}$; $=0.90$, SEE $=9.9$ $\mathrm{ml}$, respectively). Similarly, EF derived from color Doppler correlated extremely well with that measured from 2-dimensional echocardiography $(r=0.99$, SEE $=1.6 \%)$ and cineangiography $(r=$ 0.96 , SEE $=3.4 \%$ ). Thus, EF can be accurately measured from the color Doppler examination. With the addition of automatic edge-detecting al-

From the Department of Pediatrics, C.S. Mott Children's Hospital, University of Michigan Medical Center, Ann Arbor, Michigan. Manuscript received February 12, 1991; revised manuscript received and accepted May 3, 1991.

$\Lambda$ ddress for reprints: A. Rebecca Snider, MD, C.S. Mott Children's Hospital, F1331, Box 0204, 1500 E. Medical Center Drive, Ann Arbor, Michigan 48109-0204. gorithms, this technique has the potential for providing a quick and automatic on-line calculation of EF.

(Am J Cardiol 1991;68:669-673)

I $\mathrm{n}$ the management of patients with congenital and acquired heart disease, the ejection fraction (EF) is a commonly used and important index of left ventricular (LV) systolic function. Two-dimensional echocardiographic measurements of EF have correlated well with cineangiographic measurements of $\mathrm{EF}$ in children and adults. ${ }^{1-5}$ However, for the measurement of EF, 2-dimensional echocardiography has several limitations. First, the 2-dimensional echocardiographic technique is time-consuming and cumbersome, requiring either the use of an off-line analysis system or the loss of time on the echocardiographic equipment for on-line analysis. Second, with 2-dimensional echocardiography, the endocardial borders of the left ventricle are often poorly visualized, making manual tracing methods very difficult and the development of automatic edge-detection methods, thus far, not possible. Because Doppler color flow mapping allows visualization of blood flow in the heart, we hypothesized that this technique could be used to enhance the distinction between the ventricular walls and blood pool and, thus, measure the ventricular volumes and EF with greater ease and accuracy. The purpose of this study was to determine the accuracy of ventricular volumes and $\mathrm{EF}$ derived from manual tracings of the color Doppler images compared with those derived from 2-dimensional echocardiography and cineangiography.

\section{METHODS}

Patients: Eleven patients undergoing echocardiographic examination and cardiac catheterization between September 1989 and April 1990 were randomly selected for inclusion in the study. Their ages ranged from 4.5 months to 22 years (mean 11) and their weights ranged from 7.3 to $70.6 \mathrm{~kg}$ (mean 35.3). The study group included 3 patients with pulmonary valve stenosis, 3 with aortic valve stenosis, 1 with supravalvular aortic stenosis, 1 with pulmonary atresia and a ventricular septal defect, 1 with coarctation of the aorta 
after a ventricular septal defect repair, 1 with dilated cardiomyopathy and 1 with mitral stenosis with a prosthetic mitral valve. Patients with abnormal ventricular shapes that did not conform to Simpson's rule (i.e., univentricular heart, absence of the majority of the ventricular septum) were excluded from the study.

Echocardiographic examination: Two-dimensional and color Doppler examinations were performed within 24 hours of cardiac catheterization using a 128-element phased-array ultrasound system (Acuson). The echocardiographic images were obtained from the apical 4chamber and long-axis views. After recording the Bmode images, the color sector was positioned to include the entire left ventricle and exclude as much of the surrounding cardiac chambers as possible. The velocity scale of the color Doppler display was then decreased until all color Doppler signals arising from blood flow in the LV cavity were aliased and filled the chamber with

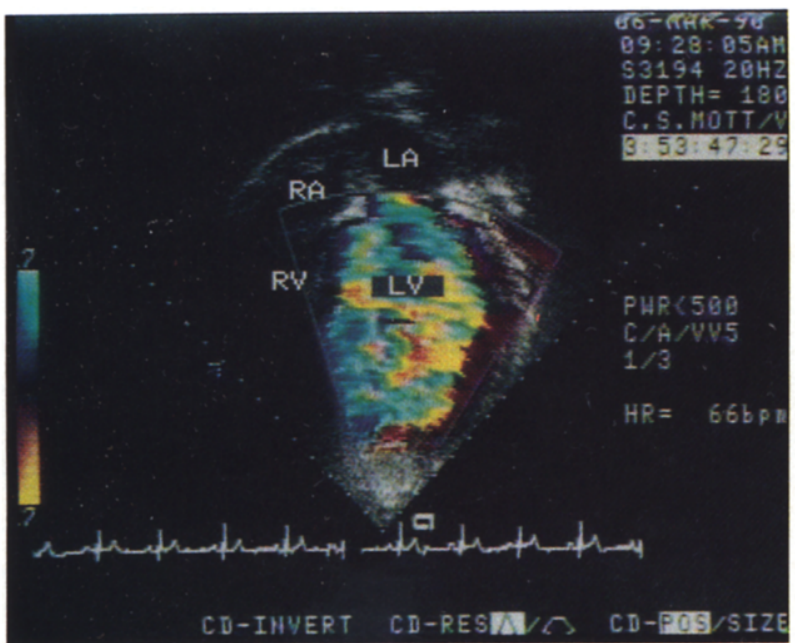

mosaic color Doppler signals, and all color Doppler signals arising from ventricular wall motion were displayed as nonaliased (pure red-blue) signals (Figure 1). Over the range of patient sizes, and heart and flow rates investigated, this required a Nyquist limit that was usually $>0.08 \mathrm{~m} / \mathrm{s}$ but always $<0.17 \mathrm{~m} / \mathrm{s}$. On the ultrasound system we used, it was necessary to use the highest possible Doppler carrier frequency in order to obtain these low Nyquist settings.

Cardiac catheterization: At the time of cardiac catheterization, biplane LV cineangiography was performed in the left anterior oblique and $30^{\circ}$ right anterior oblique projections. Although angiograms were obtained in all patients in the study, 3 patients had angiograms that could not be analyzed because of the absence of any sinus beats. The cineangiograms were transferred to $1 / 2$-inch videotape for later off-line analysis.

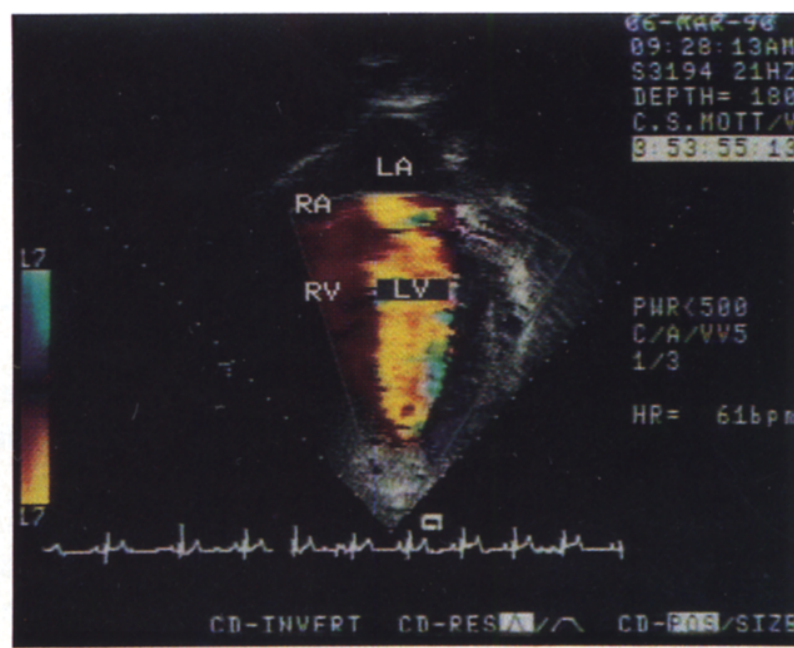

FICURE 1. Color Doppler images at end-dlastole (left) and end-systole (right) showing the technique used to determine left ventricular ejection fraction. The color sector is placed so as to include the entire left ventricle (LV), and the velocity scale has been decreased to the lowest possible setting. Note that the left ventricular cavity is filled with aliased (mosaic) color Doppler signals, whereas color Doppler signals arising from the left ventricular walls are pure red-blue (nonaliased). LA $=$ left atrium; $R A=$ right atrium; $\mathbf{R V}=$ right ventricle
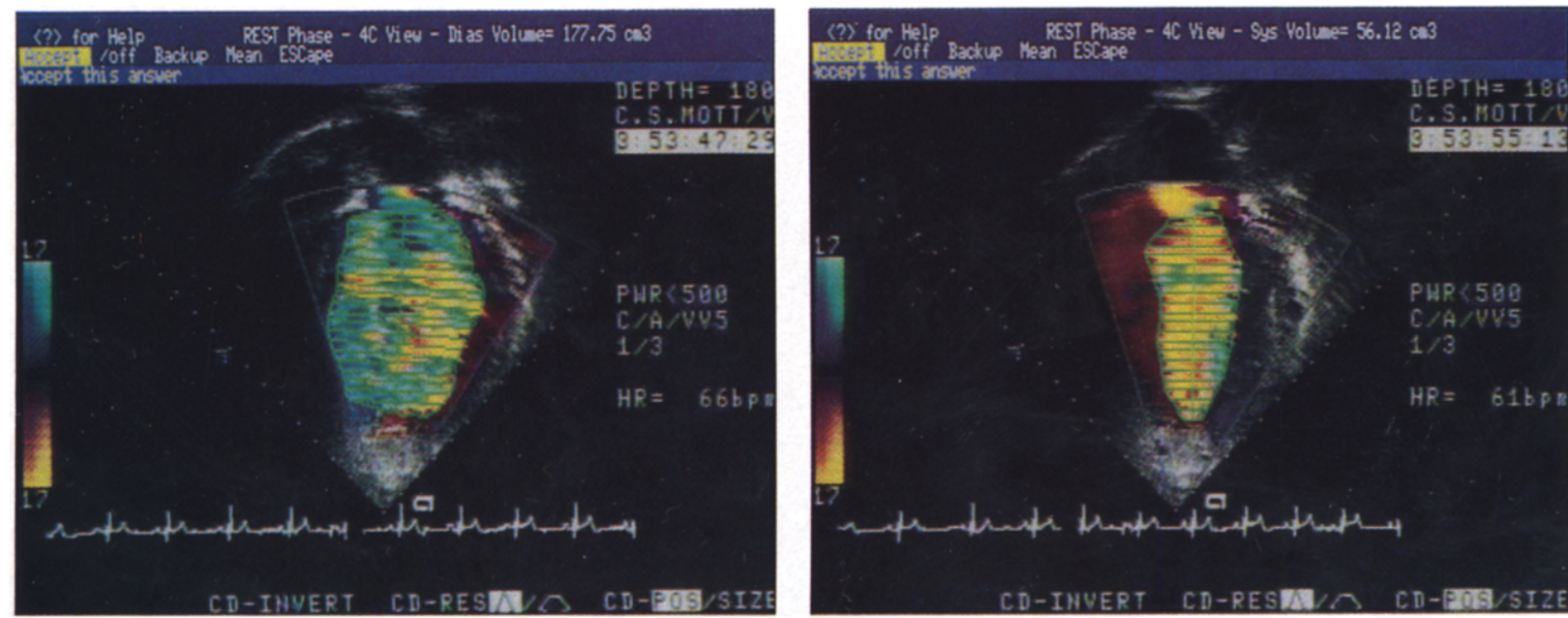

FICURE 2. Color Doppler images at end-diastole (left) and end-systole (right) showing the technique used to calculate left ventricular volumes. The border of the color Doppler signals filling the left ventricle (mosaic signals) was manually traced (green Ine). The computer program then applied a Simpson's rule algorithm to calculate left ventricular volumes and ejection fraction. 
Analysis: The echocardiograms and cineangiograms were analyzed by 2 observers who had no knowledge of the results of the other technique. All images were analyzed on a commercially available off-line analysis system equipped with a color frame grabber and software programs for biplane volume analysis (Freeland Medical System). For the color Doppler images, the outer margin of the mosaic flow area was traced manually at end-diastole and end-systole (Figure 2). The 2-dimensional and cineangiographic images were also digitized and traced along the endocardial border using the offline system.

End-diastolic and end-systolic volumes and $\mathrm{EF}$ were calculated using a Simpson's rule method and paired biplane data for each measurement. For all the echo-

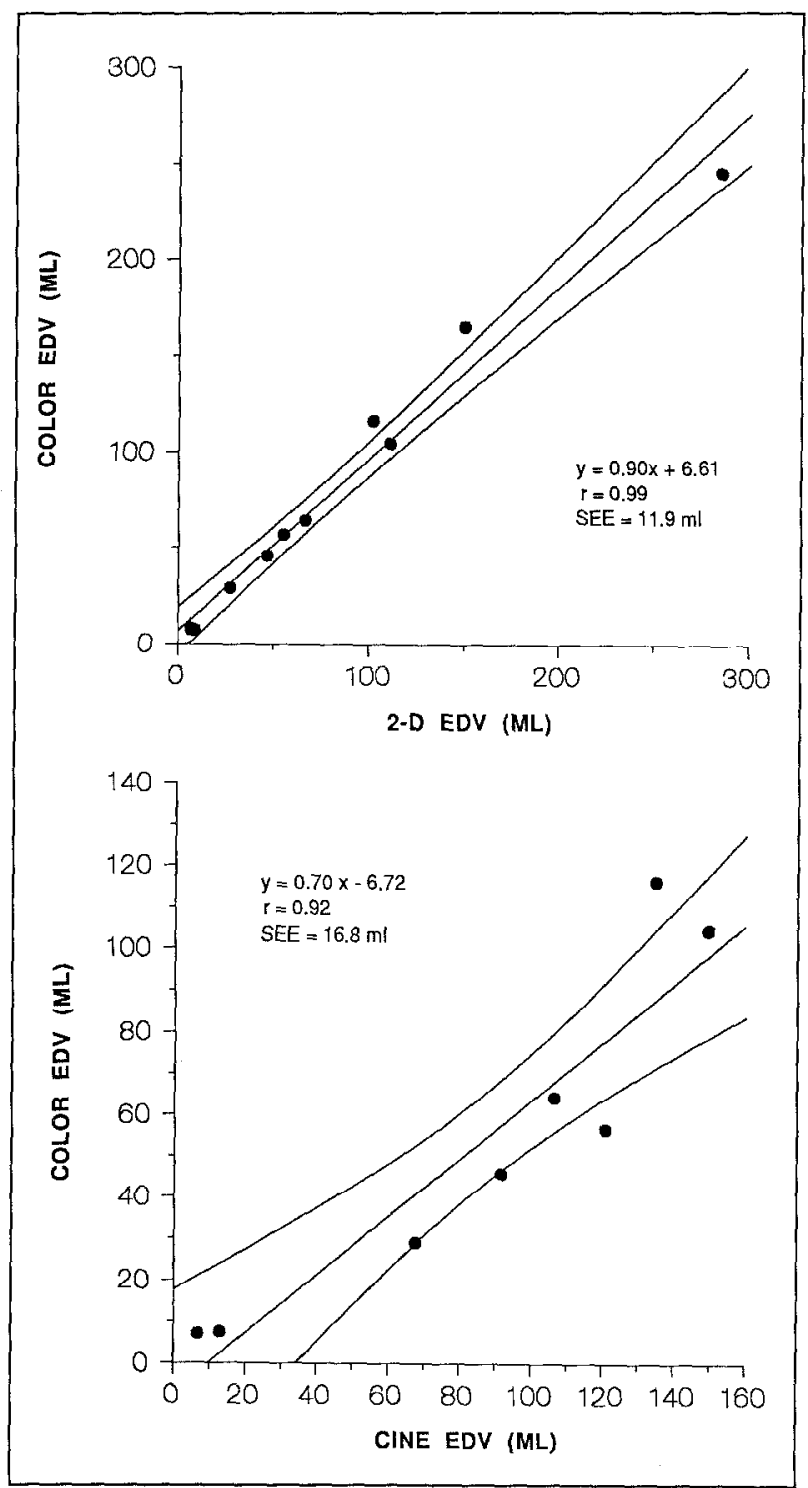

FICURE 3. Correlation between left ventricular end-diastolic volumes (EDV) measured from color Doppler and those measured from 2-dimensional (2-D) echocardiography (top) and cineangiography (CINE) (bottom). Additional lines represent the 5 and $95 \%$ confidence limits. SEE = standard error of the estimate. cardiographic and color Doppler studies, 3 cardiac cycles were measured and averaged. For the cineangiograms, 3 cardiac cycles were measured when available. With use of linear regression analysis, volumes and EF obtained from color Doppler were compared with those obtained from 2-dimensional echocardiography and cineangiography.

\section{RESULTS}

Figure 3 shows the comparisons of end-diastolic volumes measured from color Doppler with those measured from 2-dimensional echocardiography and cineangiography. For both comparisons, there was an excellent correlation $(r=0.99, \quad$ SEE $=11.9 \mathrm{ml}$, and $r=0.92, \mathrm{SEE}=16.8 \mathrm{ml}$, respectively). Although end-

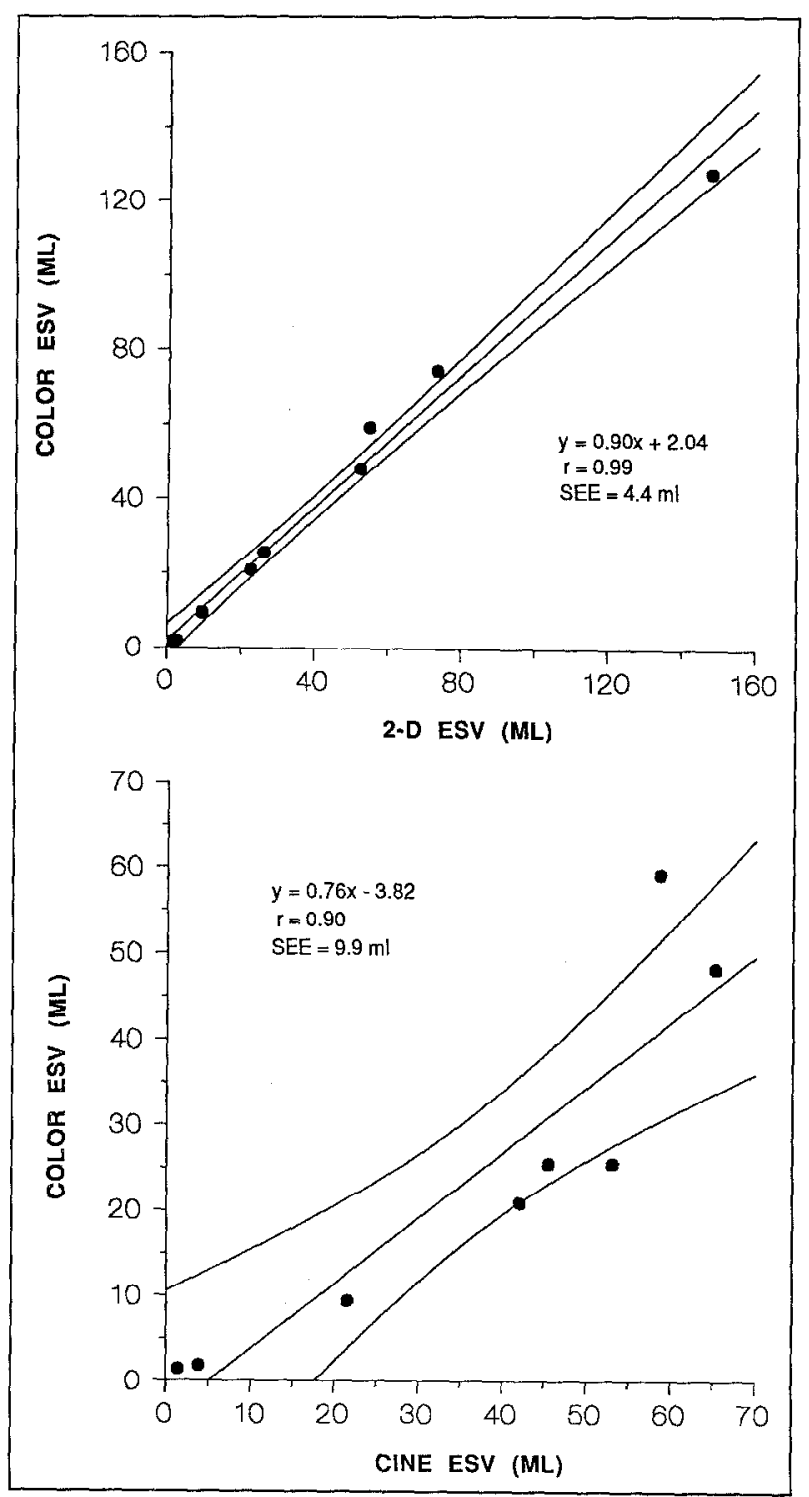

FIGURE 4. Correlation between left ventricular end-systolic volumes (ESV) measured from color Doppler and those measured from 2-dimensional (2-D) echocardiography (top) and cineangiography (CINE) (bottom). Additional lines represent the 5 and $95 \%$ confidence limits. SEE = standard error of the estimate. 
diastolic volumes obtained using color Doppler and 2dimensional echocardiography were nearly identical, the color Doppler technique tended to underestimate the end-diastolic volume obtained with cineangiography.

Similarly, end-systolic volumes measured from color Doppler were nearly identical to those measured from 2-dimensional echocardiography (Figure 4). For the 2 techniques, $r=0.99$ and $\mathrm{SEE}=4.4 \mathrm{ml}$. As with enddiastolic volumes, the end-systolic volumes obtained from color Doppler tended to underestimate those obtained from cineangiography (Figure 4). For these 2 techniques, $r=0.90$ and $\mathrm{SEE}=9.9 \mathrm{ml}$.

Figure 5 shows the excellent correlation found between EF calculated from color Doppler and EF calcu-

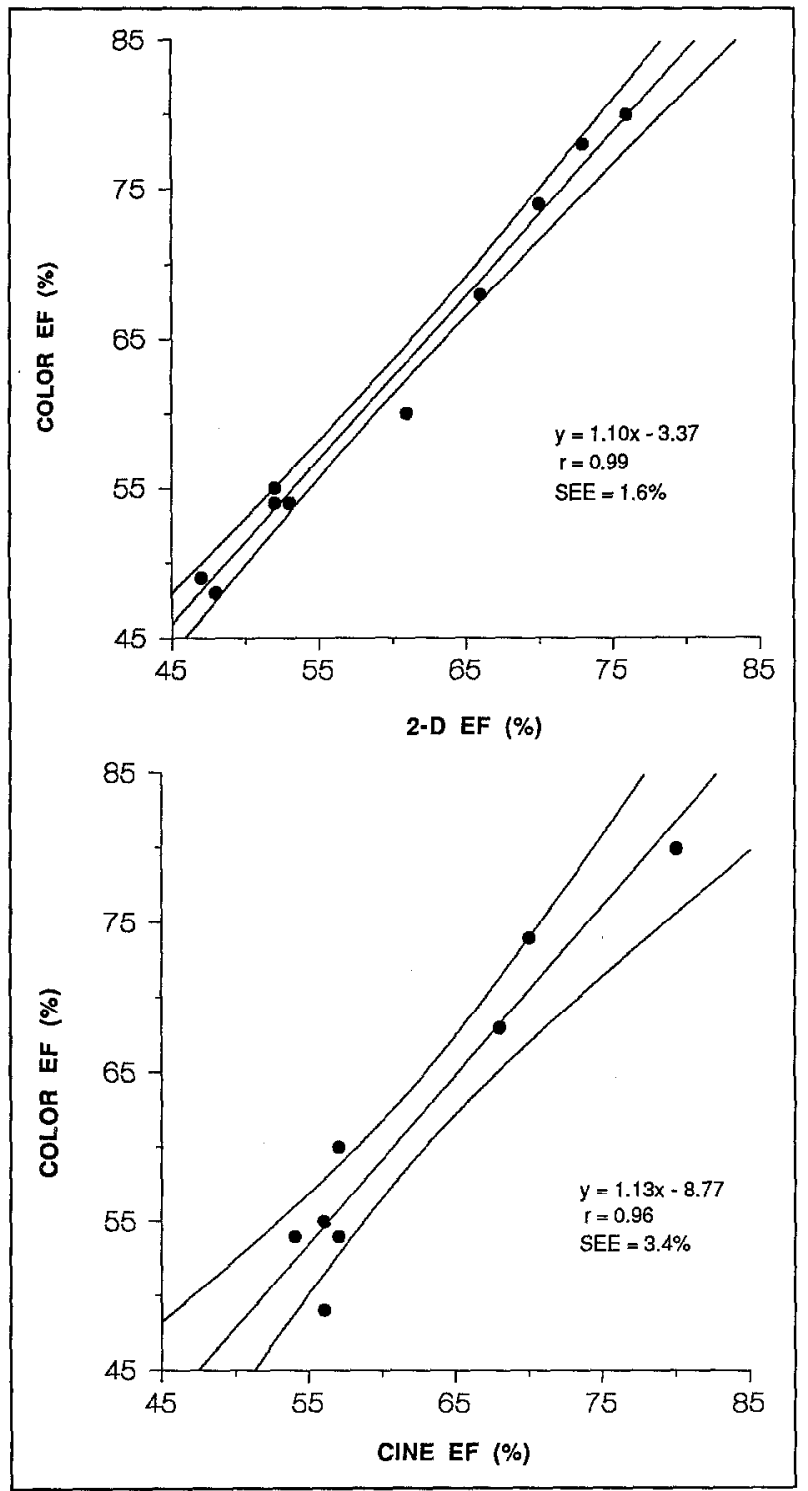

FIGURE 5. Correlation between left ventricular ejection fractions (EF) measured from color Doppler and those measured from 2-dimensional (2-D) echocardiography (top) and cineangiography (CINE) (bottom). Additional lines represent the 5 and $95 \%$ confidence limits. SEE = standard error of the estimate. lated from 2-dimensional echocardiography $(r=0.99$, $\mathrm{SEE}=1.6 \%)$ and cineangiography $(\mathrm{r}=0.96, \mathrm{SEE}=$ $3.4 \%$ ). For both comparisons, EF obtained using the color Doppler technique tended to be slightly higher.

\section{DISCUSSION}

In this study, LV volumes and EF measured from manual tracings of Doppler color flow images correlated well with those measured from 2-dimensional echocardiography and cineangiography. Color Doppler and 2-dimensional echocardiographic results were nearly identical; however, volumes measured from color Doppler tended to underestimate those obtained from cineangiography. A similar trend has been observed when 2-dimensional echocardiographic volumes have been compared with cineangiographic volumes. ${ }^{2}$ It is likely that catheterization volumes tend to be larger because of angiographic contrast filling of trabecular interspaces. The results of these comparisons highlight a limitation of the present study-i.e., the lack of a suitable reference standard to which the color Doppler data can be compared. Comparisons of echocardiographic and cineangiographic results have several "built in" sources of error that cannot usually be eliminated in the study design. Among these are lack of simultaneity of the studies and technical differences such as differences in the imaging planes, differences in sampling rates, and differences in image resolution. For future use of the color Doppler technique, it is extremely encouraging that the 2 noninvasive techniques yielded nearly identical results.

Although the 2-dimensional echocardiographic and color Doppler studies were obtained nearly simultaneously, with the same equipment, and in the same imaging planes, 1 major difference between the 2 techniques was the sampling rate or frame rate. On the 2dimensional echocardiographic examinations, sampling rates were usually between 30 and 40 frames/s; however, on the color Doppler examinations, sampling rates were often as low as 15 frames/s. This could potentially result in an inability to identify precisely end-systole and end-diastole and, thus, should be a more important source of error in examining pediatric patients with rapid heart rates. For the color Doppler technique, we recommend that the color Doppler equipment be set so that color frame rates ranging from 15 to 25 frames/s are obtained.

The patients in this study were all children or young adults with EF ranging from 47 to $80 \%$. We did not examine any technically difficult adult patients with low EF or apical aneurysms in which flow velocities may be extremely low. One anticipated problem with these adult patients is that, at the depths required to obtain their apical views, the velocity scale of the equipment may not have a low enough setting to allow alias- 
ing of color Doppler signals in the cardiac apex; or, even if the velocity scale can be set low enough, color Doppler signals from wall motion might also be aliased, causing confusion in recognizing the border between the cavity and the walls. Since this study was completed, we have successfully examined several young adult patients with dilated cardiomyopathy and EFs in the range of $20 \%$ using the color Doppler technique; however, studies in large numbers of technically difficult adult patients are necessary to determine the usefulness and limitations of the color Doppler technique in these patients. If necessary, the current Doppler equipment could be modified by the manufacturers in order to examine difficult adult patients. For example, the velocity scale and wall filters of the equipment could be modified or color Doppler power and velocity information may be combined to delineate better the boundary between the LV cavity and walls.

In patients with poor-quality 2-dimensional echocardiographic imaging, the color Doppler technique may enhance the distinction between the endocardial borders and blood pool and, thus, improve our ability to determine the boundaries of the LV cavity. In addition, the color Doppler technique may be extremely impor- tant in the future development of technology for the automatic, on-line calculation of LVEF.

Acknowledgment: We wish to thank Lyne MeridaAsmus for technical assistance and Kathlene Chmielewski, CMA, for editorial assistance in the preparation of this manuscript.

\section{REFERENCES}

1. Folland ED, Parisi $A F$, Moynihan PF, Jones DR, Feldman CL, Tow DE. Assessment of left ventricular ejection fraction and volumes by real-time, twodimensional echocardiography. A comparison of cineangiographic and radionuclide techniques. Circulation 1979;60:760-766.

2. Silverman NH, Ports TA, Snider AR, Schiller NB. Determination of left ventricular volume in children: echocardiographic and angiographic comparisons. Circulation 1980;62;62:548-557.

3. Mercier JC, DiSessa TG, Jarmakani JM, Nakanishi T, Hiraishi S, IsabelJones J, Friedman WF. Two-dimensional echocardiographic assessment of left ventricular volumes and ejection fraction in children. Circulation 1982; 65:962-969.

4. Schiller NB, Acquatella H, Ports TA, Drew D, Goerke J, Ringertz H, Silverman NH, Brundage B, Botvinick EJ, Boswell R, Carlsson E, Parmley WW. Left ventricular volume from paired biplane two-dimensional echocardiography. Cir. culation 1979;60:547-555.

5. Wahr DW, Wang YS, Schiller NB. Left ventricular volumes determined by two-dimensional echocardiography in a normal adult population. $J$ Am Coll Cardiol 1983;1:863-868. 